\title{
CHARACTERIZATIONS OF BERGMAN SPACES AND BLOCH SPACE IN THE UNIT BALL OF $\mathbf{C}^{n}$
}

\author{
CAIHENG OUYANG, WEISHENG YANG, AND RUHAN ZHAO
}

\begin{abstract}
In this paper we prove that, in the unit ball $B$ of $\mathbf{C}^{n}$, a holomorphic function $f$ is in the Bergman space $L_{a}^{p}(B), 0<p<\infty$, if and only if

$$
\int_{B}|\tilde{\nabla} f(z)|^{2}|f(z)|^{p-2}\left(1-|z|^{2}\right)^{n+1} d \lambda(z)<\infty,
$$

where $\tilde{\nabla}$ and $\lambda$ denote the invariant gradient and invariant measure on $B$, respectively. Further, we give some characterizations of Bloch functions in the unit ball $B$, including an exponential decay characterization of Bloch functions. We also give the analogous results for $\operatorname{BMOA}(\partial B)$ functions in the unit ball.
\end{abstract}

\section{INTRODUCTION}

Let $A(B)$ denote the class of holomorphic functions in the unit ball of $\mathbf{C}^{n}$. For $0<p<\infty$, the Bergman spaces $L_{a}^{p}(B)$, the Hardy spaces $H^{p}(B)$ and the Bloch space $\mathscr{B}(B)$ on the unit ball $B$ are defined respectively as

$$
\begin{gathered}
L_{a}^{p}(B)=\left\{f: f \in A(B),\|f\|_{L_{a}^{p}}^{p}=\int_{B}|f(z)|^{p} d m(z)<\infty\right\}, \\
H^{p}(B)=\left\{f: f \in A(B),\|f\|_{H^{p}}^{p}=\sup _{0<r<1} \int_{\partial B}|f(r \xi)|^{p} d \sigma(\xi)<\infty\right\}
\end{gathered}
$$

and

$$
\mathscr{B}(B)=\left\{f: f \in A(B),\|f\|_{\mathscr{B}}=\sup _{z \in B} Q_{f}(z)<\infty\right\},
$$

where $Q_{f}$ was defined by R. Timoney in [9], $d m$ is the normalized Lebesgue measure on $B$, and $d \sigma$ is the normalized Lebesgue measure on the boundary $\partial B$ of $B$.

In [8], M. Stoll proved that a holomorphic function $f$ on $B$ is in $H^{p}(B)$, $0<p<\infty$, if and only if

$$
\int_{B}|\widetilde{\nabla} f(z)|^{2}|f(z)|^{p-2}\left(1-|z|^{2}\right)^{n} d \lambda(z)<\infty,
$$

where $\tilde{\nabla}$ denotes the invariant gradient and $\lambda$ the invariant measure on $B$. Furthermore, if $f \in H^{p}(B)$, then

Received by the editors September 19, 1994.

1991 Mathematics Subject Classification. Primary 32A37. 


$$
\lim _{r \rightarrow 1}\left(1-r^{2}\right)^{n} \int_{B_{r}}|\widetilde{\nabla} f(z)|^{2}|f(z)|^{p-2} d \lambda(z)=0,
$$

where $B_{r}=\{z \in B:|z|<r\}$.

These results were first given by S. Yamashita in [11] and [12] in the unit disk of C. In [13], the results for Bergman spaces similar to that of Yamashita's were given on the unit disk $D$.

The main purpose of this paper is to obtain the analogous result for functions in the Bergman spaces $L_{a}^{p}$ on the unit ball $B$ of $C^{n}$. Furthermore, some new characterizations of Bloch space $\mathscr{B}(B)$, including an exponential decay type characterization, are given too. The main results of this paper, which are also similar to that of [13] in case $n=1$, are as follows:

Theorem 1. A holomorphic function $f$ is in $L_{a}^{p}(B), 0<p<\infty$, if and only if

$$
\int_{B}|\widetilde{\nabla} f(z)|^{2}|f(z)|^{p-2}\left(1-|z|^{2}\right)^{n+1} d \lambda(z)<\infty .
$$

Furthermore, if $f \in L_{a}^{p}(B)$, then

$$
\lim _{r \rightarrow 1}\left(1-r^{2}\right)^{n+1} \int_{B_{r}}|\widetilde{\nabla} f(z)|^{2}|f(z)|^{p-2} d \lambda(z)=0 .
$$

Theorem 2. Let $n>1, p \geq 2$; then the following quantities are equivalent:

(i) $\|f\|_{\mathscr{B}}^{p}$,

(ii) $\quad J_{2}=\sup _{a \in B} \int_{B}|\widetilde{\nabla} f(z)|^{2}|f(z)-f(a)|^{p-2}$

$$
\cdot\left(1-\left|\varphi_{a}(z)\right|^{2}\right)^{n+1}\left|\varphi_{a}(z)\right|^{-2 n+2} d \lambda(z),
$$

(iii) $J_{3}=\sup _{a \in B} \int_{B}|\widetilde{\nabla} f(z)|^{2}|f(z)-f(a)|^{p-2}[G(z, a)]^{1+\frac{1}{n}} d \lambda(z)$,

where $\varphi_{a}$ denotes the involutive automorphism of $B$ satisfying $\varphi_{a}(0)=a$, $\varphi_{a}(a)=0, \varphi_{a}\left(\varphi_{a}(z)\right)=z$, and $G(z, a)$ denotes the Green's function of $B$.

Theorem 3. Let $n>1$; then a holomorphic function $f \in \mathscr{B}(B)$ if and only if for every $a \in B$ and every $t>0$ there are positive constants $K$ and $\beta$, such that

$$
\int_{E_{a, t}}|\widetilde{\nabla} f(z)|^{2}[G(z, a)]^{1+\frac{1}{n}} d \lambda(z) \leq K e^{-\beta t},
$$

where $E_{a, t}=\{z \in B:|f(z)-f(a)|>t\}$. When $f \in \mathscr{B}, K=K_{0}\|f\|_{\mathscr{B}}^{2}$, $\beta=C /\|f\|_{\mathscr{B}}$, where $K_{0}$ and $C$ are constants depending only on $n$.

In Section 2, we first give some notations. Theorem 1 is proved in Section 3. Theorems 2 and 3 are proved in Section 4. In Section 5, we give some characterizations of $\operatorname{BMOA}(\partial B)$ which are similar to Theorems 2 and 3.

\section{NotATIONS}

For each $a \in B$, let $\varphi_{a}(z)$ denote the involutive automorphism of $B$ as given in [6] by W. Rudin. Let $\nabla f(z)=\left(\partial f / \partial z_{1}, \ldots, \partial f / \partial z_{n}\right)$ denote the complex gradient of $f$ and $R f=\sum_{j=1}^{n} z_{j}\left(\partial f / \partial z_{j}\right)$ the radial derivative of $f$. Let

$$
d \lambda(z)=\frac{n+1}{\left(1-|z|^{2}\right)^{n+1}} d m(z)
$$


then $d \lambda$ is the invariant volume measure corresponding to the Bergmen metric on $B$; that is,

$$
\int_{B} f(z) d \lambda(z)=\int_{B} f \circ \psi(z) d \lambda(z)
$$

for each $f \in L^{1}(d \lambda)$ and all $\psi \in \mathscr{M}$, the group of Möbius transformations of $B$.

For $f \in C^{2}(\Omega), \Omega$ an open subset of $B$, define

$$
\tilde{\Delta} f(z)=\frac{1}{n+1} \Delta\left(f \circ \varphi_{z}\right)(0),
$$

as in [1],

$$
\tilde{\Delta} f(z)=\frac{4}{n+1}\left(1-|z|^{2}\right) \sum_{i, j=1}^{n}\left(\delta_{i j}-z_{i} \bar{z}_{j}\right) \frac{\partial^{2} f(z)}{\partial z_{i} \partial \bar{z}_{j}} .
$$

The operator $\widetilde{\Delta}$ is invariant under $\mathscr{M}$; that is, $\widetilde{\Delta}(f \circ \psi)=(\widetilde{\Delta} f) \circ \psi$ for all $\psi \in \mathscr{M}$. See [6, Section 4.1] for details. Let $\widetilde{\nabla}$ denote the invariant gradient on $B$. Then

$$
(\widetilde{\nabla} f(z), \widetilde{\nabla} g(z))=\frac{4}{n+1}\left(1-|z|^{2}\right) \mathscr{R}\left[\sum_{i, j=1}^{n}\left(\delta_{i j}-z_{i} \bar{z}_{j}\right) \frac{\partial f}{\partial z_{i}} \frac{\partial g}{\partial \bar{z}_{j}}\right] \text {. }
$$

If $f$ is holomorphic on $B$, it is given in [8] that

$$
\widetilde{\Delta}|f|^{2}=|\widetilde{\nabla} f|^{2}=\frac{4}{n+1}\left(1-|z|^{2}\right)\left(|\nabla f|^{2}-|R f|^{2}\right) .
$$

Throughout this paper, $C$ and $C_{j}$ are constants depending only on the dimension $n . M$ is a finite number, and $M(r)$ is a finite number for a fixed $r \in(0,1] . C$ is not necessarily the same in each appearance, nor are $C_{j}, M$, $M(r)$.

For convenience, $A(f, r) \sim B(f, r)$ means that there exist constants $N_{1}$, $N_{2}, C_{1}$ and $C_{2}$, so that

$$
N_{1}+C_{1} A(f, r) \leq B(f, r) \leq N_{2}+C_{2} A(f, r),
$$

where $N_{1}, N_{2}$ may depend on $f$, but they are finite quantities for a fixed function $f$.

By [10], the invariant Green's function on $B$ is given by $G(z, a)=g\left(\varphi_{a}(z)\right)$, where

$$
g(z)=\frac{n+1}{2 n} \int_{|z|}^{1}\left(1-t^{2}\right)^{n-1} t^{-2 n+1} d t .
$$

Here we state the Green's formula for an invariant Laplacian (see [7, (92.5)]). If $\Omega$ is an open subset of $B, \bar{\Omega} \subset B$, whose boundary is good enough (in our application, $\Omega$ will be an annulus) and if $u, v$ are real-valued functions such that $u, v \in C^{2}(\Omega) \cap C^{1}(\bar{\Omega})$, then

$$
\int_{\Omega}(u \tilde{\Delta} v-v \tilde{\Delta} u) d \bar{\tau}=\int_{\partial \Omega}\left(u \frac{\partial v}{\partial \bar{n}}-v \frac{\partial u}{\partial \bar{n}}\right) d \bar{\sigma},
$$

where $\bar{\tau}$ and $\bar{\sigma}$ are the volume element on $B$ and surface area element on $\partial \Omega$ determined by the Bergman metric, and $\frac{\partial}{\partial n}$ denotes the outward normal 
differentiation along $\partial \Omega$ with respect to the Bergman metric. It is known (cf. [1]) that the volume element $\bar{\tau}$ is given by

$$
d \bar{\tau}(z)=\frac{\omega_{n}(n+1)^{n}}{2 n\left(1-|z|^{2}\right)^{n+1}} d m(z),
$$

where $\omega_{n}$ denotes the Euclidean surface area of $\partial B$ and the surface area element $\bar{\sigma}_{r}$ on $\partial B_{r}$ is given by

$$
d \bar{\sigma}_{r}(r \xi)=\frac{\omega_{n}(n+1)^{n-\frac{1}{2}} r^{2 n-1}}{\left(1-r^{2}\right)^{n}} d \sigma(\xi)
$$

\section{Proof of THEOREM 1}

To prove Theorem 1, for $\varepsilon>0$, let

$$
v_{\varepsilon}(z)=\left(|f(z)|^{2}+\varepsilon\right)^{p / 2}, \quad 0<p<\infty ;
$$

then $v_{\varepsilon} \in C^{\infty}$. Since $\tilde{\Delta} g=0$ on $B \backslash\{0\}$ and $g=g(r)$ on $\partial B_{r}=\{z \in B$ : $|z|=r\}$, using Green's formula with $u=g-g(r), v=v_{\varepsilon}$ and $\Omega=\{z \in B$ : $\delta<|z|<r\}$, we can conclude

$$
\begin{aligned}
\int_{\delta<|z|<r} & (g-g(r)) \tilde{\Delta} v_{\varepsilon} d \bar{\tau} \\
= & -\int_{\partial B_{r}} v_{\varepsilon} \frac{\partial g}{\partial \bar{n}} d \bar{\sigma}_{r}-\left[(g(\delta)-g(r)) \int_{\partial B_{\delta}} \frac{\partial v_{\varepsilon}}{\partial \bar{n}} d \bar{\sigma}-\int_{\partial B_{\delta}} v_{\varepsilon} \frac{\partial g}{\partial \bar{n}} d \bar{\sigma}_{\delta}\right] .
\end{aligned}
$$

Because $\frac{\partial v_{\varepsilon}}{\partial n}$ is bounded on $\partial B_{\delta}, g(\delta) \delta^{2 n-1} \rightarrow 0(\delta \rightarrow 0)$ and $g$ is integrable near $0(\delta \neq 0)$, taking the limit $\delta \rightarrow 0$, we get

$$
\int_{B_{r}}(g-g(r)) \tilde{\Delta} v_{\varepsilon} d \lambda=\int_{\partial B} v_{\varepsilon}(r \xi) d \sigma(\xi)-v_{\varepsilon}(0) .
$$

Let

$$
f_{p}^{\#}(z)=\frac{p^{2}}{4}|f(z)|^{p-2}|\tilde{\nabla} f(z)|^{2},
$$

by [8], for $0<p<\infty$; when $\varepsilon \rightarrow 0$,

$$
\widetilde{\Delta} v_{\varepsilon}(z) \rightarrow f_{p}^{\#}(z) \text { a.e. on } B \text {. }
$$

For a fixed $r$, from (1) and by the monotone convergence theorem, we have

$$
\begin{aligned}
\lim _{\varepsilon \rightarrow 0} \int_{B_{r}}(g-g(r)) \tilde{\Delta} v_{\varepsilon} d \lambda & =\lim _{\varepsilon \rightarrow 0}\left(\int_{\partial B} v_{\varepsilon}(r \xi) d \sigma(\xi)-v_{\varepsilon}(0)\right) \\
& =\int_{\partial B}|f(r \xi)|^{p} d \sigma(\xi)-|f(0)|^{p} .
\end{aligned}
$$

By (2) and the Fatou Lemma

$$
\begin{aligned}
\int_{B_{r}}(g-g(r)) f_{p}^{\#} d \lambda & =\int_{B_{r}} \frac{\lim }{\varepsilon \rightarrow 0}(g-g(r)) \tilde{\Delta} v_{\varepsilon} d \lambda \\
& \leq \frac{\lim }{\varepsilon \rightarrow 0} \int_{B_{r}}(g-g(r)) \tilde{\Delta} v_{\varepsilon} d \lambda \\
& =\int_{\partial B}|f(r \xi)|^{p} d \sigma(\xi)-|f(0)|^{p}=M(r)<\infty
\end{aligned}
$$


thus, $(g-g(r)) f_{p}^{\#}$ is integrable on $B_{r}$ with respect to $d \lambda$.

As $0<p<2$, for a fixed $r$, by [8], $\widetilde{\Delta} v_{\varepsilon}(z) \leq \frac{2}{p} f_{p}^{*}(z)$, a.e. on $B$, and thus

$$
(g-g(r)) \tilde{\Delta} v_{\varepsilon} \leq(g-g(r)) \frac{2}{p} f_{p}^{\#}, \quad \text { a.e. on } B .
$$

As $p \geq 2$, for $\varepsilon \in(0,1]$

$$
\widetilde{\Delta} v_{\varepsilon} \leq M(r)<\infty, \quad \text { on } B_{r},
$$

and thus

$$
(g-g(r)) \tilde{\Delta} v_{\varepsilon} \leq M(r)(g-g(r))
$$

(here $g-g(r)$ is obviously integrable on $B_{r}$ ).

Using the dominated convergence theorem with both $0<p<2$ and $p \geq 2$ and from (2), we get

$$
\begin{aligned}
\int_{B_{r}}(g-g(r)) f_{p}^{\#} d \lambda & =\lim _{\varepsilon \rightarrow 0} \int_{B_{r}}(g-g(r)) \tilde{\Delta} v_{\varepsilon} d \lambda \\
& =\int_{\partial B}|f(r \xi)|^{p} d \sigma(\xi)-|f(0)|^{p} .
\end{aligned}
$$

Let

$$
\chi_{|z|}(t)= \begin{cases}1, & t>|z| \\ 0, & \text { otherwise }\end{cases}
$$

then the left side of $(3)$ is

$$
\begin{aligned}
\int_{B_{r}}( & (z)-g(r)) f_{p}^{\#}(z) d \lambda(z) \\
& =\int_{B_{r}} f_{p}^{\#}(z) d \lambda(z)\left(\frac{n+1}{2 n} \int_{|z|}^{r} \frac{\left(1-t^{2}\right)^{n-1}}{t^{2 n-1}} d t\right) \\
& =\frac{n+1}{2 n} \int_{B_{r}} f_{p}^{\#}(z) d \lambda(z) \int_{0}^{r} \frac{\left(1-t^{2}\right)^{n-1}}{t^{2 n-1}} \chi_{|z|}(t) d t \\
& =\frac{n+1}{2 n} \int_{0}^{r} \frac{\left(1-t^{2}\right)^{n-1}}{t^{2 n-1}} d t \int_{B_{l}} f_{p}^{\#}(z) d \lambda(z) .
\end{aligned}
$$

Obviously, the end of (4)

$$
\begin{aligned}
& \frac{n+1}{2 n} \int_{0}^{r} \frac{\left(1-t^{2}\right)^{n-1}}{t^{2 n-1}} d t \int_{B_{t}} f_{p}^{\#}(z) d \lambda(z) \\
& \quad \geq \frac{n+1}{2 n} r^{-2 n+1} \int_{0}^{r}\left(1-t^{2}\right)^{n-1} d t \int_{B_{t}} f_{p}^{\#}(z) d \lambda(z) .
\end{aligned}
$$

On the other hand, for $0<r<1$, there exists a positive integer $k$, so that $1 / 2^{k}<r \leq 1 / 2^{k-1}$; then 
4306

CAIHENG OUYANG, WEISHENG YANG, AND RUHAN ZHAO

$$
\begin{aligned}
& \frac{n+1}{2 n} \int_{0}^{r} \frac{\left(1-t^{2}\right)^{n-1}}{t^{2 n-1}}\left(\int_{B_{t}} f_{p}^{\#}(z) d \lambda(z)\right) d t \\
& \quad=\frac{n+1}{2 n}\left(\int_{0}^{\frac{1}{2^{k}}}+\int_{\frac{1}{2^{k}}}^{r}\right) \frac{\left(1-t^{2}\right)^{n-1}}{t^{2 n-1}}\left(\int_{B_{t}} f_{p}^{\#}(z) d \lambda(z)\right) d t \\
& \quad \leq \frac{n+1}{2 n} \int_{0}^{\frac{1}{2}} \frac{\left(1-t^{2}\right)^{n-1}}{t^{2 n-1}}\left(\int_{B_{t}} f_{p}^{\#}(z) d \lambda(z)\right) d t
\end{aligned}
$$

$$
\begin{aligned}
& +\frac{n+1}{2 n} 2^{k(2 n-1)}\left(\frac{2^{-(k-1)}}{r}\right)^{2 n-1} \int_{0}^{r}\left(1-t^{2}\right)^{n-1}\left(\int_{B_{t}} f_{p}^{\#}(z) d \lambda(z)\right) d t \\
= & \frac{n+1}{2 n} \int_{0}^{\frac{1}{2}} \frac{\left(1-t^{2}\right)^{n-1}}{t^{2 n-1}}\left(\int_{B_{t}} f_{p}^{\#}(z) d \lambda(z)\right) d t \\
& +\frac{n+1}{2 n} 2^{2 n-1} r^{-2 n+1} \int_{0}^{r}\left(1-t^{2}\right)^{n-1}\left(\int_{B_{t}} f_{p}^{\#}(z) d \lambda(z)\right) d t \\
= & I_{1}+I_{2} .
\end{aligned}
$$

By (4) and (3),

$$
\begin{aligned}
I_{1} & =\int_{B_{\frac{1}{2}}}\left(g(z)-g\left(\frac{1}{2}\right)\right) f_{p}^{\#}(z) d \lambda(z) \\
& =\int_{\partial B}\left|f\left(\frac{1}{2} \xi\right)\right|^{p} d \sigma(\xi)-|f(0)|^{p}<\infty .
\end{aligned}
$$

Thus, it follows from (4), (5) and (6) that

$$
\int_{B_{r}}(g(z)-g(r)) f_{p}^{\#}(z) d \lambda(z) \sim r^{-2 n+1} \int_{0}^{r}\left(1-t^{2}\right)^{n-1}\left(\int_{B_{t}} f_{p}^{\#}(z) d \lambda(z)\right) d t .
$$

Moreover, by (3), we have

$$
\int_{\partial B}|f(r \xi)|^{p} d \sigma(\xi) \sim r^{-2 n+1} \int_{0}^{r}\left(1-t^{2}\right)^{n-1}\left(\int_{B_{t}} f_{p}^{\#}(z) d \lambda(z)\right) d t .
$$

Hence

$$
\begin{aligned}
\|f\|_{L_{a}^{p}}^{p} & =2 n \int_{0}^{1} r^{2 n-1} d r \int_{\partial B}|f(r \xi)|^{p} d \sigma(\xi) \\
& \sim \int_{0}^{1} d r\left(\int_{0}^{r}\left(1-t^{2}\right)^{n-1} d t \int_{B_{t}} f_{p}^{\#}(z) d \lambda(z)\right) \\
& =\int_{0}^{1}\left(1-t^{2}\right)^{n-1} d t \int_{B_{t}} f_{p}^{\#}(z) d \lambda(z) \int_{t}^{1} d r \\
& \sim \int_{0}^{1}\left(1-t^{2}\right)^{n} d t \int_{B_{t}} f_{p}^{\#}(z) d \lambda(z) \\
& =\int_{B} f_{p}^{\#}(z) d \lambda(z) \int_{|z|}^{1}\left(1-t^{2}\right)^{n} d t \\
& \sim \int_{B}\left(1-|z|^{2}\right)^{n+1} f_{p}^{\#}(z) d \lambda(z) \\
& \sim \int_{B}|\widetilde{\nabla} f(z)|^{2}|f(z)|^{p-2}\left(1-|z|^{2}\right)^{n+1} d \lambda(z),
\end{aligned}
$$


and thus

$$
f \in L_{a}^{p}(B) \Leftrightarrow \int_{B}|\widetilde{\nabla} f(z)|^{2}|f(z)|^{p-2}\left(1-|z|^{2}\right)^{n+1} d \lambda(z)<\infty .
$$

That is the main part of Theorem 1 .

When $f \in L_{a}^{p}(B)$, by the above result,

$$
\begin{aligned}
& \int_{0}^{1} \int_{B_{t}}|\widetilde{\nabla} f(z)|^{2}|f(z)|^{p-2}\left(1-|z|^{2}\right)^{n} d \lambda(z) d t \\
& \quad=\int_{B} \int_{|z|}^{1}|\widetilde{\nabla} f(z)|^{2}|f(z)|^{p-2}\left(1-|z|^{2}\right)^{n} d t d \lambda(z) \\
& \quad \leq \int_{B}|\widetilde{\nabla} f(z)|^{2}|f(z)|^{p-2}\left(1-|z|^{2}\right)^{n+1} d \lambda(z)<\infty,
\end{aligned}
$$

and thus

$$
\lim _{r \rightarrow 1} \int_{r}^{1} \int_{B_{t}}|\widetilde{\nabla} f(z)|^{2}|f(z)|^{p-2}\left(1-|z|^{2}\right)^{n} d \lambda(z) d t=0 .
$$

Furthermore

$$
\begin{aligned}
(1- & \left.r^{2}\right)^{n+1} \int_{B_{r}}|\widetilde{\nabla} f(z)|^{2}|f(z)|^{p-2} d \lambda(z) \\
& \leq 2(1-r) \int_{B_{r}}|\widetilde{\nabla} f(z)|^{2}|f(z)|^{p-2}\left(1-|z|^{2}\right)^{n} d \lambda(z) \\
& \leq 2 \int_{r}^{1} \int_{B_{t}}|\widetilde{\nabla} f(z)|^{2}|f(z)|^{p-2}\left(1-|z|^{2}\right)^{n} d \lambda(z) d t .
\end{aligned}
$$

By (8) and (9), we conclude

$$
\lim _{r \rightarrow 1}\left(1-r^{2}\right)^{n+1} \int_{B_{r}}|\widetilde{\nabla} f(z)|^{2}|f(z)|^{p-2} d \lambda(z)=0 .
$$

That is the last part of Theorem 1.

Remark 1. From (7) we can get another proof of Theorem 1 of [8]. In fact, letting $r \rightarrow 1$ in (7), we get

$$
\begin{aligned}
\|f\|_{H^{p}}^{p} & \sim \int_{0}^{1}\left(1-t^{2}\right)^{n-1} d t \int_{B_{t}} f_{p}^{\#}(z) d \lambda(z) \\
& =\int_{B} f_{p}^{\#}(z) d \lambda(z) \int_{|z|}^{1}\left(1-t^{2}\right)^{n-1} d t \\
& \sim \int_{B}|\widetilde{\nabla} f(z)|^{2}|f(z)|^{p-2}\left(1-|z|^{2}\right)^{n} d \lambda(z) .
\end{aligned}
$$

Remark 2. Theorem 2 in [8] can be concluded from (3). Taking the limit $r \rightarrow 1$ on two sides of (3), using the monotone convergence theorem, we get

$$
\|f\|_{H^{p}}^{p}=|f(0)|^{p}+\frac{p^{2}}{4} \int_{B}|\widetilde{\nabla} f(z)|^{2}|f(z)|^{p-2} g(z) d \lambda(z) .
$$

This is equivalent to the result of Theorem 2 of [8]. 


\section{Characterizations of Bloch space in the UNit ball}

Lemma 1. Let $n \geq 2$ be an integer; then there are constants $C_{1}$ and $C_{2}$ such that, for all $z \in B \backslash\{0\}$,

$$
C_{1}\left(1-|z|^{2}\right)^{n}|z|^{-2(n-1)} \leq g(z) \leq C_{2}\left(1-|z|^{2}\right)^{n}|z|^{-2(n-1)},
$$

where

$$
g(z)=\frac{n+1}{2 n} \int_{|z|}^{1} r^{-2 n+1}\left(1-r^{2}\right)^{n-1} d r .
$$

Proof. It is easy to see that

$$
\lim _{|z| \rightarrow 1} \frac{g(z)}{\left(1-|z|^{2}\right)^{n}|z|^{-2(n-1)}}=\frac{n+1}{4 n^{2}},
$$

and

$$
\lim _{|z| \rightarrow 0} \frac{g(z)}{\left(1-|z|^{2}\right)^{n}|z|^{-2(n-1)}}=\frac{n+1}{4 n(n-1)} .
$$

The result of Lemma 1 comes by the continuity of $g(z),(11)$ and (12).

Proof of Theorem 2. Replacing $f$ in (3) by $f_{a}=f \circ \varphi_{a}(\cdot)-f \circ \varphi_{a}(0)$, we get

$$
\begin{aligned}
& \frac{p^{2}}{4} \int_{B_{r}}\left|\widetilde{\nabla} f_{a}(w)\right|^{2}\left|f_{a}(w)\right|^{p-2}(g(w)-g(r)) d \lambda(w) \\
& \quad=\int_{\partial B}\left|f_{a}(r \zeta)\right|^{p} d \sigma(\zeta) .
\end{aligned}
$$

Therefore

$$
\begin{aligned}
\frac{4}{p^{2}}\left\|f_{a}\right\|_{L_{a}^{p}}^{p} & =\frac{4}{p^{2}} \int_{B}\left|f_{a}(z)\right|^{p} d m(z)=\frac{8 n}{p^{2}} \int_{0}^{1} r^{2 n-1} d r \int_{\partial B}\left|f_{a}(r \zeta)\right|^{p} d \sigma(\zeta) \\
& =2 n \int_{0}^{1} r^{2 n-1} d r \int_{B_{r}}\left|\widetilde{\nabla} f_{a}(w)\right|^{2}\left|f_{a}(w)\right|^{p-2}(g(w)-g(r)) d \lambda(w) \\
& \leq 2 n \int_{0}^{1} d r \int_{B_{r}}\left|\widetilde{\nabla} f_{a}(w)\right|^{2}\left|f_{a}(w)\right|^{p-2}(g(w)-g(r)) d \lambda(w) \\
& =(n+1) \int_{0}^{1} d r \int_{0}^{r} \frac{\left(1-t^{2}\right)^{n-1}}{t^{2 n-1}} d t \int_{B_{t}}\left|\widetilde{\nabla} f_{a}(w)\right|^{2}\left|f_{a}(w)\right|^{p-2} d \lambda(w) \\
& =(n+1) \int_{0}^{1} \frac{\left(1-t^{2}\right)^{n-1}}{t^{2 n-1}} d t \int_{t}^{1} d r \int_{B_{t}}\left|\widetilde{\nabla} f_{a}(w)\right|^{2}\left|f_{a}(w)\right|^{p-2} d \lambda(w) \\
& \leq(n+1) \int_{0}^{1} \frac{\left(1-t^{2}\right)^{n}}{t^{2 n-1}} d t \int_{B_{t}}\left|\widetilde{\nabla} f_{a}(w)\right|^{2}\left|f_{a}(w)\right|^{p-2} d \lambda(w) \\
& =(n+1) \int_{B}\left|\widetilde{\nabla} f_{a}(w)\right|^{2}\left|f_{a}(w)\right|^{p-2} d \lambda(w) \int_{|w|}^{1} \frac{\left(1-t^{2}\right)^{n}}{t^{2 n-1}} d t \\
& \leq 2 n \int_{B}\left|\widetilde{\nabla} f_{a}(w)\right|^{2}\left|f_{a}(w)\right|^{p-2}\left(1-|w|^{2}\right) g(w) d \lambda(w) \\
& \leq C \int_{B}\left|\widetilde{\nabla} f_{a}(w)\right|^{2}\left|f_{a}(w)\right|^{p-2}\left(1-|w|^{2}\right)^{n+1}|w|^{-2 n+2} d \lambda(w) .
\end{aligned}
$$


The last inequality is given by Lemma 1 . Letting $\varphi_{a}(w)=z$, we can find

$$
\begin{aligned}
\left\|f_{a}\right\|_{L_{a}^{p}}^{p} \leq C \int_{B}|\tilde{\nabla} f(z)|^{2}|f(z)-f(a)|^{p-2} \\
\cdot\left(1-\left|\varphi_{a}(z)\right|^{2}\right)^{n+1}\left|\varphi_{a}(z)\right|^{-2 n+2} d \lambda(z) .
\end{aligned}
$$

Thus we have

$$
\sup _{a \in B}\left\|f_{a}\right\|_{L_{a}^{p}}^{p} \leq C J_{2}
$$

By Theorem 4.7 in [9],

$$
\|f\|_{\mathscr{B}} \leq C\|f\|_{X}
$$

where $\|f\|_{X}=\sup _{z \in B}|\nabla f(z)|\left(1-|z|^{2}\right)$. By the lemma in [5].

$$
\|f\|_{X} \leq c \sup _{a \in B}\left\|f_{a}\right\|_{L_{a}^{p}}
$$

Therefore

$$
\|f\|_{\mathscr{B}}^{P} \leq C J_{2} .
$$

Because $\left|\varphi_{a}(z)\right|<1$ for $z, a \in B$, we know

$$
\left|\varphi_{a}(z)\right|^{-2 n+2} \leq\left|\varphi_{a}(z)\right|^{-2\left(n-\frac{1}{n}\right)} .
$$

By Lemma 1 and $G(z, a)=g\left(\varphi_{a}(z)\right)$,

$$
\left(1-\left|\varphi_{a}(z)\right|^{2}\right)^{n+1}\left|\varphi_{a}(z)\right|^{-2\left(n-\frac{1}{n}\right)} \leq C(G(z, a))^{1+\frac{1}{n}} .
$$

Hence

$$
\begin{aligned}
J_{2} \leq & \sup _{a \in B} \int_{B}|\tilde{\nabla} f(z)|^{2}|f(z)-f(a)|^{p-2} \\
& \leq C \sup _{a \in B} \int_{B}|\tilde{\nabla} f(z)|^{2}|f(z)-f(a)|^{p-2}(G(z, a))^{1+\frac{1}{n}} d \lambda(z) \\
& =C J_{3} .
\end{aligned}
$$

Now let $\|f\|_{\mathscr{B}}<\infty$. By Theorem 4.7 in [9],

$$
|\nabla f(z)|\left(1-|z|^{2}\right) \leq C_{1}\|f\|_{\mathscr{B}} .
$$

Thus by Lemma 2.2 in [2],

$$
\left|\nabla_{T} f(z)\right|\left(1-|z|^{2}\right)^{\frac{1}{2}} \leq C_{2}\|f\|_{\mathscr{B}},
$$

where $\nabla_{T} f$ is the complex tangential gradient of $f$. Hence by the proof of Theorem 2.4 in [2],

$$
\begin{aligned}
|\widetilde{\nabla} f(z)|^{2} & =\widetilde{\Delta}|f|^{2}(z) \\
& \leq 4\left(1-|z|^{2}\right)^{2}|\nabla f(z)|^{2}+4\left(1-|z|^{2}\right)\left|\nabla_{T} f(z)\right|^{2} \\
& \leq C\|f\|_{\mathscr{B}}^{2} .
\end{aligned}
$$


From this and Lemma 1,

$$
\begin{aligned}
J_{3}(a) & =\int_{B}|\widetilde{\nabla} f(z)|^{2}|f(z)-f(a)|^{p-2}(G(z, a))^{1+\frac{1}{n}} d \lambda(z) \\
& \leq C\|f\|_{\mathscr{B}}^{2} \int_{B}\left|f \circ \varphi_{a}(w)-f \circ \varphi_{a}(0)\right|^{p-2}(g(w))^{1+\frac{1}{n}} \\
& \cdot\left(1-|w|^{2}\right)^{-n-1} d m(w) \\
& \leq C\|f\|_{\mathscr{B}}^{2} \int_{B}\left|f_{a}(w)\right|^{p-2}|w|^{\frac{-2\left(n^{2}-1\right)}{n}} d m(w) .
\end{aligned}
$$

When $p=2$

$$
\begin{aligned}
J_{3}(a) & \leq C\|f\|_{\mathscr{B}}^{2} \int_{B}|w|^{\frac{-2\left(n^{2}-1\right)}{n}} d m(w) \\
& =2 n C\|f\|_{\mathscr{B}}^{2} \int_{0}^{1} r^{2 n-1-\frac{2\left(n^{2}-1\right)}{n}} d r \\
& =2 n C\|f\|_{\mathscr{B}}^{2} \int_{0}^{1} r^{\frac{2}{n}-1} d r=n^{2} C\|f\|_{\mathscr{B}}^{2} .
\end{aligned}
$$

When $p>2$, let $\alpha=\max \left(n^{2}+1, \frac{1}{p-2}\right)$; then it is easy to know that

$$
\left(\int_{B}|w|^{\frac{-2\left(n^{2}-1\right)}{n} \frac{\alpha}{\alpha-1}} d m(w)\right)^{1-\frac{1}{\alpha}}=M<\infty .
$$

By the Lemma in [5],

$$
\left(\int_{B}\left|f_{a}(w)\right|^{(p-2) \alpha} d m(w)\right)^{1 / \alpha} \leq C(\Gamma((p-2) \alpha+1))^{1 / \alpha}\|f\|_{\mathscr{B}}^{p-2} .
$$

Thus, by (15), using the Hölder inequality

$$
\begin{aligned}
J_{3}(a) \leq & C\|f\|_{\mathscr{B}}^{2}\left(\int_{B}\left|f_{a}(w)\right|^{(p-2) \alpha} d m(w)\right)^{\frac{1}{\alpha}} \\
& \cdot\left(\int_{B}|w|^{\frac{-2\left(n^{2}-1\right)}{n} \frac{\alpha}{\alpha-1}} d m(w)\right)^{1-\frac{1}{\alpha}} \\
\leq & C M(\Gamma((p-2) \alpha+1))^{\frac{1}{\alpha}}\|f\|_{\mathscr{B}}^{p} .
\end{aligned}
$$

By (16) and (17), for $p \geq 2$,

$$
J_{3}=\sup _{a \in B} J_{3}(a) \leq C(\Gamma((p-2) \alpha+1))^{1 / \alpha}\|f\|_{\mathscr{B}}^{p} .
$$

By (13), (14) and (18), the quantities $\|f\|_{\mathscr{B}}^{p}, J_{2}$ and $J_{3}$ are equivalent. The proof of Theorem 2 is complete.

Remark 3. It is authors' belief that the results of Theorem 2 should hold for all $p$ 's, that is, also for $0<p<2$. In this case more delicate techniques seem to be needed.

Proof of Theorem 3. First, let $f \in \mathscr{B}$. For each integer $k>0$, let $\alpha=n^{2}+1$ in (18), then we get

$$
\begin{aligned}
I_{k+2}(a) & =\int_{B}|\widetilde{\nabla} f(z)|^{2}|f(z)-f(a)|^{k}(G(z, a))^{1+\frac{1}{n}} d \lambda(z) \\
& \leq C\left(\Gamma\left(k\left(n^{2}+1\right)+1\right)\right)^{\frac{1}{n^{2}+1}}\|f\|_{\mathscr{B}}^{k+2} .
\end{aligned}
$$


It is easy to see that $\left(\Gamma\left(k\left(n^{2}+1\right)+1\right)\right)^{\frac{1}{n^{2}+1}} \leq\left(n^{2}+1\right)^{k} k !$. Hence

$$
I_{k+2}(a) \leq C\left(n^{2}+1\right)^{k} k !\|f\|_{\mathscr{B}}^{k+2} .
$$

Note that, when $k=0$, the above inequality is also valid by (16). Taking a constant $\tau, 0<\tau<\frac{1}{n^{2}+1}$, then if we set $\beta=\tau /\|f\|_{\mathscr{B}}$, we get

$$
\begin{aligned}
& e^{\beta t} \int_{E_{a, t}}|\widetilde{\nabla} f(z)|^{2}(G(z, a))^{1+\frac{1}{n}} d \lambda(z) \\
& \quad \leq \int_{E_{a, t}}|\widetilde{\nabla} f(z)|^{2} e^{\beta|f(z)-f(a)|}(G(z, a))^{1+\frac{1}{n}} d \lambda(z) \\
& \quad \leq \sum_{k=0}^{\infty} \frac{\beta^{k}}{k !} I_{k+2}(a) \leq C \sum_{k=0}^{\infty} \frac{\beta^{k}}{k !}\left(n^{2}+1\right)^{k} k !\|f\|_{\mathscr{B}}^{k+2} \\
& \quad=C\|f\|_{\mathscr{B}}^{2} \sum_{k=0}^{\infty}\left(\left(n^{2}+1\right) \tau\right)^{k}=K<\infty,
\end{aligned}
$$

where $K=K_{0}\|f\|_{\mathscr{B}}^{2}, K_{0}$ is an absolute constant. Hence

$$
\int_{E_{a, t}}|\widetilde{\nabla} f(z)|^{2}(G(z, a))^{1+\frac{1}{n}} d \lambda(z) \leq K e^{-\beta t} .
$$

Conversely, let $f$ satisfy (19); then

$$
\int_{0}^{\infty} d t \int_{E_{a, t}}|\widetilde{\nabla} f(z)|^{2}(G(z, a))^{1+\frac{1}{n}} d \lambda(z) \leq K \int_{0}^{\infty} e^{-\beta t} d t=\frac{K}{\beta}<\infty .
$$

But

$$
\begin{aligned}
\int_{0}^{\infty} & d t \int_{E_{a, t}}|\widetilde{\nabla} f(z)|^{2}(G(z, a))^{1+\frac{1}{n}} d \lambda(z) \\
& =\int_{B}|\widetilde{\nabla} f(z)|^{2}(G(z, a))^{1+\frac{1}{n}}\left(\int_{0}^{|f(z)-f(a)|} d t\right) d \lambda(z) \\
& =\int_{B}|\widetilde{\nabla} f(z)|^{2}|f(z)-f(a)|(G(z, a))^{1+\frac{1}{n}} d \lambda(z)
\end{aligned}
$$

So we get

$$
\sup _{a \in B} \int_{B}|\widetilde{\nabla} f(z)|^{2}|f(z)-f(a)|(G(z, a))^{1+\frac{1}{n}} d \lambda(z) \leq \frac{K}{\beta}<\infty .
$$

By Theorem 2 with $p=3$, we know that $f \in \mathscr{B}(B)$. The proof is complete.

\section{Characterizations of BMOA in the UNit Ball}

Let $f \in H^{1}(B)$, the Hardy space in the unit ball of $\mathbf{C}^{n}$. We say that $f \in \operatorname{BMOA}(\partial B)$ if its radial limit function $f^{*}$ is a function of bounded mean oscillations on $\partial B$ with respect to nonisotropic balls generated by the nonisotropic metric $\rho(\zeta, \eta)=|1-\langle\zeta, \eta\rangle|^{1 / 2}$ on $\partial B$. See [3] for details.

Let $f_{a}=f \circ \varphi_{a}(\cdot)-f \circ \varphi_{a}(0)$. In [4], Ouyang proved that a holomorphic function $f \in \operatorname{BMOA}(\partial B)$ if and only if

$$
\sup _{a \in B}\left\|f_{a}\right\|_{H^{p}}^{p}<\infty
$$


Furthermore, he proved that if $f \in \operatorname{BMOA}(\partial B)$, then

$$
\sup _{a \in B}\left\|f_{a}\right\|_{H^{p}}^{p} \leq \frac{K \Gamma(p+1)}{C^{p}}\|f\|_{* *}^{p}<\infty
$$

where

$$
\|f\|_{* *}=\sup _{a \in B}\left\|f_{a}\right\|_{H^{1}} .
$$

Now replacing $f$ in (10) with $f_{a}$, we get

$$
\begin{aligned}
\left\|f_{a}\right\|_{H^{p}}^{p} & =\frac{p^{2}}{4} \int_{B}\left|\tilde{\nabla} f_{a}(w)\right|^{2}\left|f \circ \varphi_{a}(w)-f \circ \varphi_{a}(0)\right|^{p-2} g(w) d \lambda(w) \\
& =\frac{p^{2}}{4} \int_{B}|\tilde{\nabla} f(z)|^{2}|f(z)-f(a)|^{p-2} G(z, a) d \lambda(z) .
\end{aligned}
$$

By (20), (21) and (22), we get the following

Proposition 1. For $0<p<\infty$, a holomorphic function $f \in \operatorname{BMOA}(\partial B)$ if and only if

$$
\sup _{a \in B} \int_{B}|\widetilde{\nabla} f(z)|^{2}|f(z)-f(a)|^{p-2} G(z, a) d \lambda(z)<\infty .
$$

Moreover, if $f \in \operatorname{BMOA}(\partial B)$, we have

$$
\sup _{a \in B} \int_{B}|\widetilde{\nabla} f(z)|^{2}|f(z)-f(a)|^{p-2} G(z, a) d \lambda(z) \leq \frac{K \Gamma(p+1)}{C^{p}}\|f\|_{* *}^{p} .
$$

Remark 4. When $p=2$, the above result was proved by J. S. Choa and B. R. Choe (see [1, Theorem A]).

Using (23) and a similar method of the proof of Theorem 3, we can obtain an exponential decay characterization of $\operatorname{BMOA}(\partial B)$ as follows.

Theorem 4. $A$ holomorphic function $f \in \operatorname{BMOA}(\partial B)$ if and only if for every $a \in B$ and every $t>0$,

$$
\int_{E_{a, t}}|\widetilde{\nabla} f(z)|^{2}(G(z, a)) d \lambda(z) \leq K e^{-\beta t}
$$

where $E_{a, t}=\{z \in B:|f(z)-f(a)|>t\}$, and $K, \beta>0$ are constants. When $f \in \operatorname{BMOA}(\partial B), K=K_{0}\|f\|_{* *}^{2}, \beta=C /\|f\|_{* *}$, where $K_{0}$ and $C$ are absolute constants.

\section{REFERENCES}

1. J. S. Choa and B. R. Choe, A Littlewood-Paley type identity and a characterization of BMOA, Complex Variables 17 (1991), 15-23.

2. J. S. Choa, H. O. Kim and Y. Y. Park, A Bergman-Carleson measure characterization of Bloch functions in the unit ball of $\mathbf{C}^{n}$, Bull. Korean Math. Soc. 29 (1992), 285-293.

3. R. Coifman, R. Rochberg and G. Weiss, Factorization theorems for Hardy spaces in several variables, Ann. of Math. (2) 103 (1976), 611-635.

4. C. H. Ouyang, Some classes of functions with exponential decay in the unit ball of $\mathbf{C}^{n}$, Publ. Res. Inst. Math. Sci. 25 (1989), 263-277.

5. An extension theorem for Bloch functions in the unit ball, Acta Math. Sci. 10 (1990), 455-461. (Chinese)

6. W. Rudin, Function theory in the unit ball of $\mathbf{C}^{n}$, Springer-Verlag, New York, 1980. 
7. I. Sokolnikoff, Tensor analysis, Wiley, New York, 1964.

8. M. Stoll, A characterization of Hardy spaces on the unit ball of $\mathbf{C}^{n}$, J. London Math. Soc. (2) 48 (1993), 126-136.

9. R. Timoney, Bloch functions in several complex variables. I, Bull. London Math. Soc. 12 (1980), 241-267.

10. D. Ullrich, Radial limits of $\mathscr{M}$-subharmonic functions, Trans. Amer. Math. Soc. 292 (1985), 501-518.

11. S. Yamashita, Criteria for functions to be Hardy class $H^{p}$, Proc. Amer. Math. Soc. 75 (1979), 69-72.

12. _ Holomorphic functions and area integrals, Boll. Un. Math. Ital. A6 (1982), 115-120.

13. R. H. Zhao, Characterizations of weighted Bergman spaces and Bloch space (to appear).

Wuhan Institute of Mathematical Sciences, Academia Sinica, P. O. Box 71007, Wuhan 430071, People's Republic of China 